\title{
GMR: An Attractive Resistance
}

Magnetoresistance was first discovered by Lord Kelvin. But he didn't have the technology to exploit it. Today, 141 years later, the effect, its clever younger brother giant magnetoresistance and pioneering studies of magnetic thin-film structures could soon enable very high density magnetic data storage and computer memory, by IBM science writer Michael Ross

\section{PRIZE WINNERS \\ Albert Fert, Peter Grünberg and Stuart Parkin, whose work this article describes, won this year's Hewlett- Packard Europhysics Prize}

It hasn't had the huge public splash of hightemperature superconductivity, but another recently discovered phenomenon in solid state physics has also attracted worldwide scientific interest.

The effect is called 'giant magnetoresistance', or GMR. It is hardly a household name today, but GMR may within the next few years enable dramatic increases in magnetic data storage density. The unusual and largely unexpected nature of GMR has also spurred significant interest worldwide in the science of thin-film magnetism.

What is so new and exciting about GMR? Consider that since the prolific Victorian scientist William Thomson (Lord Kelvin) discovered in 1856 that the electrical resistance of a piece of iron increased by ' $1 / 3000$ ' when subjected to a magnetic field, magnetoresistance (MR) has usually been a modest effect. Typically, the electrical resistance of a material placed in a magnetic field increases by less than 5 per cent at room temperature and by at most 30 per cent at $4.2 \mathrm{~K}$.

But in recent years, scores of new thinfilm 'multilayer' structures having resistance decreases in magnetic fields with MRs of greater than ten per cent at room temperature have been discovered. One sputter-deposited multilayer of alternating thin films of copper and a cobalt/5-per cent-iron alloy, made last year by Stuart S.P. Parkin's group at IBM's Almaden Research Center in San Jose, California, exhibits the largest MR ever measured in a metallic

What is GMR? Turn to page 116 and a guide for those innocent of solid state matters... material at room temperature (110 percent). At low temperature (4.2k), the same multilayer exhibits 220 percent, which ties with the record obtained in an MBE-grown $\mathrm{Fe} / \mathrm{Cr}$ multilayer at $1.2 \mathrm{~K}$ by the group of Yvan Bruynseraede at the Catholic University of Leuven in Belgium. Hence the name giant magnetoresistance.

If used in a magnetic sensor, GMR materials can produce a stronger, more easily detected signal than conventional MR materials. That would allow designers to increase hard disk data storage capacities by using much smaller bits than is now possible. But many of the multilayers with the highest GMR effects require very strong magnetic fields hundreds to thousands of oersteds (the Earth's magnetic field is about 0.5 oersted) - before they exhibit significant GMR. Any practical sensor for magnetic recording must respond to much weaker fields -25 oersteds or less.

Typical of early results in any emerging field of science, there are conflicting and at times incomplete explanations for some data. But it is clear that by studying how these multilayer and spin-valve structures work, scientists are greatly advancing their scientific understanding of the fundamental interactions of magnetism and electrical current in materials.

\section{Direction is the Key}

GMR has been found in thin-film and granular structures having very smooth interfaces between magnetic and nonmagnetic materials. In these structures, the key feature is the relative direction of the magnetization between the various discrete magnetic regions. GMR is seen when the relative magnetization directions of neighbouring magnetic regions change in response to an external magnetic field. In multilayer structures, the resistance is greatest when each magnetic layer's orientation is exactly opposite, or antiparallel, to its neighbours and is least when all the layers' magnetizations are oriented in the same, or parallel, direction. In their experiments, scientists measure the resistance along the planes as they vary the strength of an external field, which changes the magnetization directions of the layers.

Although many people had studied the magnetic properties of thin-film multilayers for decades, no one had ever noticed this type of magnetoresistance until the late 1980 s. As with many scientific achievements, the road to success is long, winding and loaded with collaboration, rivalry, inspiration and lots of hard work.

Peter Grünberg of KFA Jülich in Germany made the first experimental moves. After 4 years of studying dipolar dynamic coupling of ferromagnetic films across nonmagnetic spacers, he thought his next step should be to see if an indirect coupling via the electrons in metallic interlayers could also be detected. A similar interaction was already known from dilute magnetic alloys and named the RKKY interaction after the theoreticians Ruderman, Kittel, Kasuya and Yosida. While he was on sabbatical at the Argonne National Laboratory in Illinois, Grünberg's hosts Mervin Brodsky and Harvy Sowers used molecular beam epitaxy (MBE) techniques for making atomically precise

\section{Dollars and Bits by the Billion}

The hard-disk drive industry was worth US\$46 billion in 1996, and is growing rapidly. IBM predicts that giant magnetoresistance will have a big impact on hard drives, allowing bit densities to continue to grow at 60 per cent a year, writes Toby Chapman.

IBM's love of launching record-breaking hard drives was satisfied once more in July when the Deskstar 8, designed for desktop personal computers, became available. Smaller than a paperback novel it holds $8.4 \mathrm{~Gb}$, around 8 times larger than hard drives in most desktop computers in use. Basically, its components send out stronger signals than current drives, so its magnetoresistive heads can read in a smaller space - the Deskstar 8 stores 270 million bits per square centimetre.

But the giant magnetoresistance effect is not only for hard drives, it can be used wherever a sensitive detector is needed, such as credit card machines, or magnetic tape drives. However, Philips research in Holland is not as convinced as IBM is about the future of GMR. Philips launched in June DigaMax, a new tape storage system that uses MR heads, but GMR is only one of many choices for future heads. If Philips does choose this route, however, it will increase data storage capacity by a factor of ten. 
superlattices to make him single-crystallike thin-film sandwiches of antiferromagnetic chromium between layers of ferromagnetic and lattice-matched iron, grown on a sodium chloride crystal. By the end of his sabbatical, Grünberg had measured antiferromagnetic coupling of the iron films across the chromium when the chromium layer was about 10 Angstroms thick. But the measurement was not reproducible enough to be worthy of publication. 'I then learned that Gary Prinz of the Naval Research Laboratory in Washington D.C. had shown that iron grows well on gallium arsenide substrates,' Grünberg says. 'We changed to this substrate and the coupling effects were reproducible.'

Grünberg's 1986 paper on the antiferromagnetic coupling in the $\mathrm{Fe} / \mathrm{Cr} / \mathrm{Fe}$ trilayers stimulated Albert Fert at the University of Paris, Orsay, to look at the electrical resistance properties of $\mathrm{Fe} / \mathrm{Cr}$ multilayers, a stack of alternating thin films. For more than a decade, Fert and colleagues had been studying the theory of spindependent conduction of electrons in ferromagnetic materials. Available MBE tools and Grünberg's inspiration gave Fert's group ample reason to test many of the theories they had helped develop.

In 1988 and at about the same time, both scientists had significant results: Grünberg reported a large 6 percent MR effect for his trilayer at $4.2 \mathrm{~K}$ in a 1,000-oersted magnetic field, and Fert disclosed an astonishing 50 per cent MR effect in a multilayer 'superlattice' of alternating layers of iron and chromium at $4.2 \mathrm{~K}$ in a very strong 2000 -oersted field.

'I was very surprised to see such high MR values,' Fert recalls. 'I thought that some unavoidable structural disorder or interface roughness would moderate any spin-enhanced MR effect in these systems.'

Before he published his results and while he was preparing for a teaching assignment at the University of Cologne on the science and applications of layered magnetic structures, Grünberg realized the potential practical importance of GMR sensors. He quickly arranged for a patent to be filed in Europe, Japan and the US covering various aspects of antiparallelpolarized components in magnetic recording heads.

In July 1988, Parkin and a number of other IBM scientists attended the 12th ICMFS (International Colloquium on Magnetic Films and Surfaces) in Le Creusot, France and heard Fert describe his GMR results. Already noted for his work with organic and high temperature superconductors, the English-born and educated Parkin was at the conference to learn about magnetic thin films, which were of growing interest at IBM-Almaden, the research center where IBM invented the first commercial hard-disk drive in the mid-1950s.

But it wasn't until he received Fert's full technical paper a few months later that Parkin became deeply interested in the subject. 'It did seem like a curiously large effect,' Parkin recalls. Almaden's MBE machine was busy with other work at the time, so Parkin decided to see if he could make some GMR samples in a new sputtering apparatus he had designed for such work.

'Frankly, I didn't think the sputtered films would be good enough,' Parkin says. 'Like everyone else, I believed only MBE could produce the needed uniformly smooth angstrom-thick films,' Parkin says.

\section{Films in Less than Three Hours}

'But to my great surprise, we saw the GMR in our first batch of sputtered iron/chromium multilayers,' he adds. And since the GMRs measured in the first sputtered samples were nearly as high as those in Fert's MBE-grown ones, Parkin had shown clearly that single-crystal quality superlattices were not required to study the causes of GMR. This achievement effectively opened this new field to the many scientists and laboratories with access to high-quality sputtering equipment.

It turned out that the high-throughput design of Parkin's computer-controlled sputtering apparatus was perfectly suited for making the dozens of different samples with various film compositions and thicknesses needed to track down clues to the causes of GMR. 'With its 20-sample carousel, the machine can produce 20 entirely different multilayer films under identical conditions in less than three hours,' says Parkin's technical assistant Kevin P. Roche. 'MBE is a much more timeconsuming process; it would take several weeks to make the same 20 multilayer films.'

To test the explanations for the GMR effect offered by Fert, Parkin and Roche first made multilayer films of element combinations that Fert's theoretical models predicted should have high GMR when alloyed: cobalt/chromium, iron/vanadium and iron/manganese. None showed any GMR.

'We were quite perplexed,' Parkin says. 'So we decided to investigate combinations in which both the magnetic and nonmagnetic layers had the same crystal structure. Indeed, we then found a small GMR in our first sample, a cobalt/ruthenium multilayer,' Parkin recalls. By varying the magnetic material, nonmagnetic metallic spacer layer material and the film thicknesses of each, they have measured the properties of more than

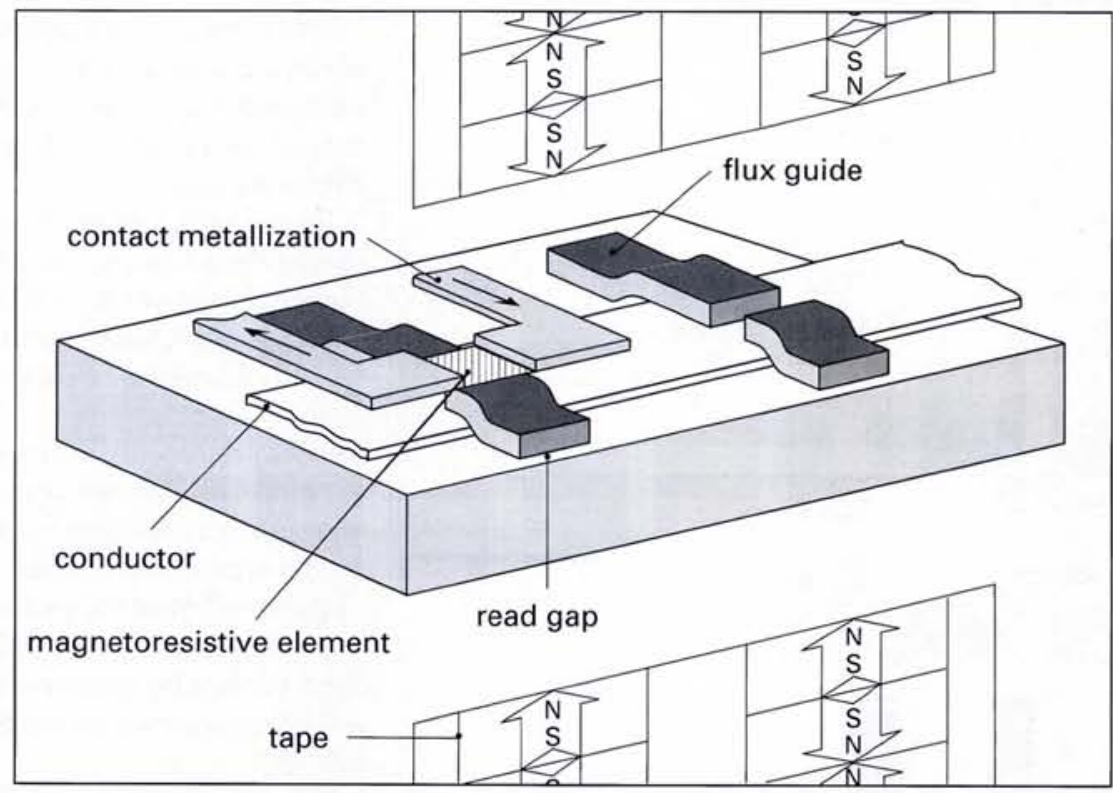

One design of a magnetoresistive head for digital data storage on magnetic tape. It is very similar to that used in Philips's latest tape storage technology, DigaMax, which uses magnetoresistive heads, and it is suitable for the application of GMR. The magnetoresistive element
(MRE) is connected by a pair of leads over which the sense current is applied and output voltage measured. The magnetically active parts of the head are the MRE, the ferate substrate and magnetic flux guides. The conductor is to provide a dc bias field during operation. 
30000 different multilayer structures over the past seven years. Many of their findings were surprising and remain the subject of worldwide theoretical interest. (See text on unexpected results.)

Because scientists studying the fundamentals of GMR need to control precisely the relative magnetization of the neighbouring magnetic layers, there are advantages to studying the simplest system: a sandwich of two magnetic layers separated by a single non-magnetic, conducting film. That's exactly the approach that Parkin and colleagues took shortly after Parkin showed that sputtered films could exhibit GMR. The team added a layer of iron-manganese, a strongly antiferromagnetic alloy, to one side of this sandwich to hold constant, or pin, the magnetic orientation of the adjacent magnetic film. Pinning permits an external field to rotate the magnetization of the other magnetic layer precisely, even if the magnetic layers are not naturally aligned antiparallel. This type of thin-film sandwich was dubbed a spin valve because of its similarity to the magnetic-valve sandwiches first studied by M. Julliere and John Slonczewski at IBM's T.J. Watson Research Center in New York in 1975.

The spin valve's versatility is needed when investigating candidates for low-field magnetic sensors. The high fields required by Grünberg's and Fert's original crystalline GMR structures and Parkin's polycrystalline GMR structures were the consequence of the strong antiferromagnetic coupling between the multilayers' closely spaced magnetic layers. The spin valve's spacer layer is deliberately made thick enough so the magnetic layers are very weakly coupled to each other. With only feeble coupling forces to overcome, modest external fields could now rotate the magnetization of these spin valves' unpinned magnetic layers and create GMR.
While an MR effect of less than 2 per cent in thin films of permalloy (an iron-nickel alloy) can make sensitive MR recording heads, spin-valve sensors are expected to perform much better. Indeed, in 1994 IBM scientists demonstrated an experimental spin-valve recording head that was the most sensitive to date with a signal of one thousandth of a volt for every micron of data track width. Spin-valve head technologies have been discussed by several disk-drive manufacturers. GMR heads are expected to enable manufacturers to continue the torrid 60 per cent annual increase in data density that the industry has maintained since IBM introduced the first MR head in 1991. Using GMR heads, 10 billion bits per square inch - quadruple the density of today's most advanced disk drive - is expected to be achieved by the turn of the century.

The challenge for nearly all applications is to find a

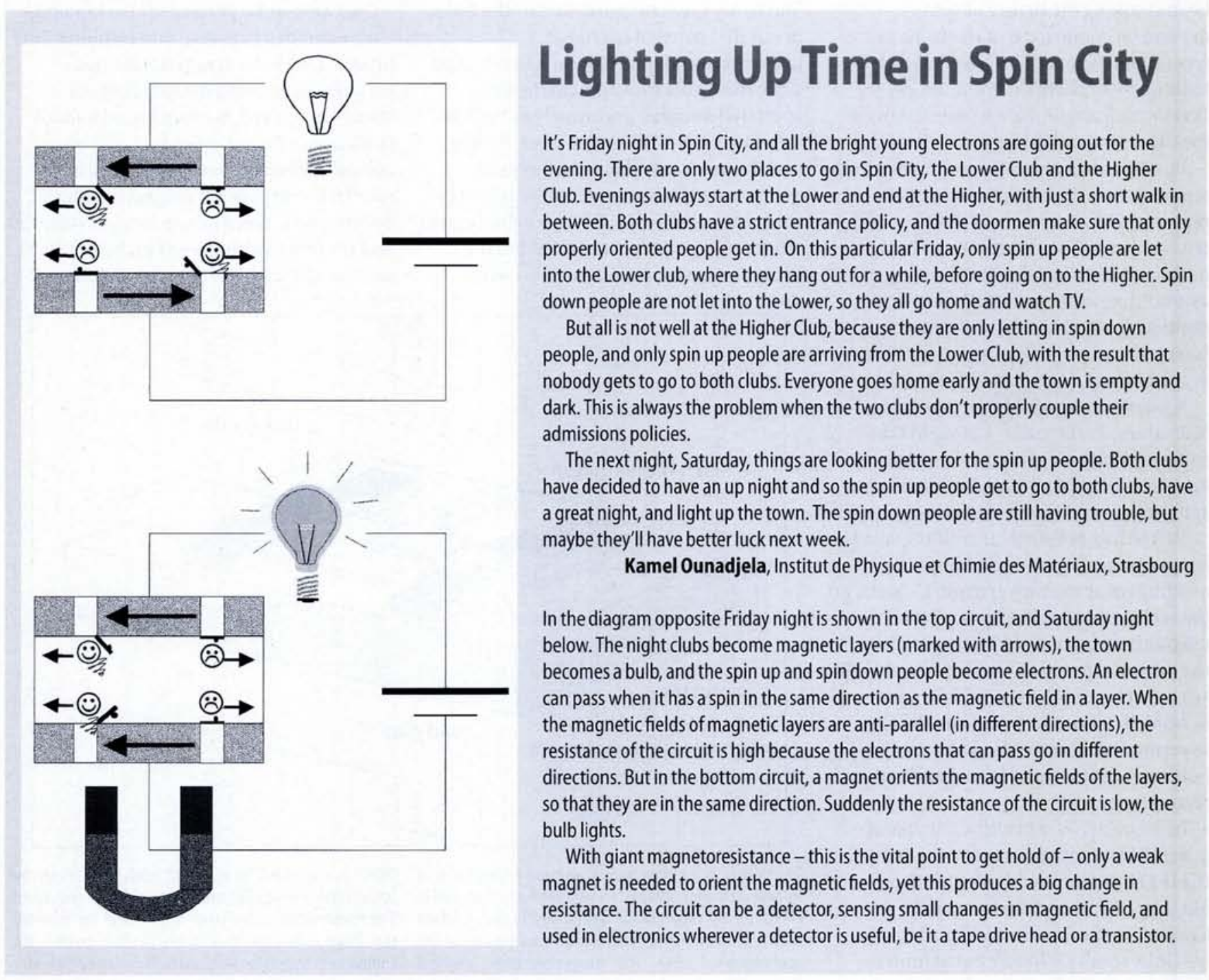




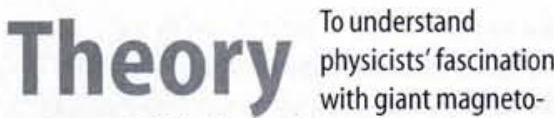

resistance (GMR), it is useful to compare it with 'regular,' or anisotropic, MR. Most electrons in an atom are confined to orbits around the atomic nucleus. Metals and, to a lesser extent, semiconductors also have additional, unbound 'conduction' electrons that are free to move throughout the material. In contrast, insulators contain few, if any, conduction electrons. In its simplest terms, electricity is the flow of these conduction electrons through a material.

Along their way, the conduction electrons may collide with obstacles within the material and change direction. Such events, called scattering, cause electrical resistance. With the notable exception of superconductors, all materials - even good electrical conductors - offer some resistance to the free flow of conduction electrons. Obstacles can include the boundaries (or interfaces) between different materials or defects in the bulk material, such as vacancies, irregularities or substituted atoms, either intentional (dopants and alloys) or unintentional (impurities).

Other obstacles are more quantum-mechanical or wavelike in character: vibrations of the crystal lattice (phonons) or of the electron spins in magnetic materials (magnons), for example. The more obstacles a material contains, the greater its scattering and resulting resistance will be. The relative scattering power of each type of obstacle may vary widely with each element, environment and temperature.

MR results when an external magnetic field increases the scattering within the material. MR is usually expressed as the ratio of the change in resistance to the resistance itself. Because resistance decreases with decreasing temperature in most metals, MR values are usually higher at low temperature, even if the actual resistance change due to the magnetic field remains constant.

While all metals show some MR effect, substantial MR at room temperature usually occurs only in magnetic metals. This is due to 'spin-orbit' coupling, the interaction of the conduction electrons' spin with the orbital paths they follow around atomic nuclei. A sufficiently strong external magnetic field aligns these electron spins and, consequently, the orbits of all the magnetic atoms in a sample. The increase in a material's resistance is anisotropic because it depends on the direction of the electrical current relative to this external field.

The chief source of GMR is 'spin-dependent' scattering. Depending on the magnetization direction, a single-domain magnetic metal layer will scatter electrons with either 'up' spins or 'down' spins, but not to the same extent. Thus, structures in which the magnetic thin films (separated by nonmagnetic metal layers) are oriented in parallel alignment have about the same electrical resistance as bulk material, irrespective of any external field because only half of the conduction electronsthose of only one spin - will be scattered heavily. However, if the magnetizations of the thin magnetic films in these structures are oriented to be antiparallel, the electrical resistance is much higher because all of the conduction electrons will be

\section{Unext sateres Unexpect namb-n ed Results mosponet layer, by the}

next. This GMR effect is greatest when the spacer layer is thin enough so any electron passing through it is rarely scattered before reaching the next magnetic layer.

The study of giant magnetoresistance quickly yielded some unusual and unpredicted behaviour that challenges our understanding of the fundamental interactions of electricity and magnetism at the atomic level.

In many magnetic/nonmagnetic metal multilayers, there are coupling forces between the magnetic layers that try to align their magnetization directions to be either parallel or antiparallel. This coupling between the magnetic layers is a fundamental property of any multilayer structure and varies with the composition and thickness of its magnetic and nonmagnetic layers. Any external field applied to such a structure tries to align all the layers to be parallel to itself. When the magnetic layers are naturally aligned in parallel, they respond to an external field in unison. Since the relative magnetization of the layers remains the same, there is no change in resistance and thus no GMR.

Layers aligned naturally antiparallel will resist the external field with a 'coupling strength' that is somewhat analogous to the force encountered when twisting a coil spring. A sufficiently strong external field can align the layers to be parallel, but when it is removed, the structure snaps back to its initial antiparallel alignment.

When Stuart S.P. Parkin's group at IBM's Almaden Research Center in California tested the various multilayers they had made with different thicknesses of the nonmagnetic'spacer' layer, they expected to find a steady decrease in coupling strength as the spacer thickness increased.

'Instead, we found something much more interesting,' Parkin recalls. The coupling strength rose and fell dramatically, although the peak magnitudes did decrease along expected values.

'At first, we thought that perhaps we had a problem with our equipment,' Parkin says. 'But we found the oscillations in coupling strength were totally reproducible. We tested a series of iron/chromium multilayers again and found clear evidence that their GMR also oscillated as the chromium spacer layer thicknesses increased.'

As he marched through the periodic table, eventually testing more than 100 different combinations of elements, Parkin found a number of common threads: The oscillation in coupling strength occurs as the spacer layer thickness increases in all systems tested; for polycrystalline multilayers, the period of the coupling strength oscillation is about the same (at about 9 to 11 angstroms) for all spacer-layer materials, with the exception of antiferromagnetic chromium, which has a 20 -angstrom oscillation period; the coupling strength between the magnetic layers for a given thickness of the nonmagnetic transition metal spacer material varies consistently and systematically across the periodic table. The coupling strength is greatest for the $3 \mathrm{~d}$ metals (vanadium, chromium and copper), followed by the $4 \mathrm{~d}$ metals (niobium, molybdenum, ruthenium and rhodium), then $5 \mathrm{~d}$ metals (tantalum, tungsten, rhenium and iridium). Within these groups, the coupling strength also increases with atomic number.

The oscillations Parkin discovered are particularly interesting because they were not predicted beforehand. Theorists in the 1960 s had calculated the properties of such multilayer structures and predicted oscillations with a 2angstrom period due to fluctuations in electronspin density.

'That the long-period oscillations were first seen on sputtered samples made the result almost suspicious,' says Peter Grünberg of KFA Jülich in Germany, who had been looking for - and not seeing - the 2-angstrom oscillations in molecularbeam-epitaxy (MBE)-grown samples. But after developing a 'wedged interlayer technique' for making MBE-quality samples with precisely and continually varying spacer-layer thicknesses, Grünberg reproduced and confirmed the longperiod oscillations Parkin had discovered.

'It's not intuitively obvious what electron property is controlling this oscillation,' says Douglas Mills, a physics professor at University of CaliforniaIrvine. As a graduate student at University of California-Berkeley, Mills in 1965 helped prove theoretically that the 2-angstrom oscillations should occur at perfectly formed magnetic/nonmagnetic metal interfaces. 'I think the 10-angstrom oscillation may have something to do with the nonideal character of the interface,' Mills adds. 'I see it as a fascinating question.'

The oscillations in both coupling strength and GMR can be explained if the magnetic layers spontaneously orient themselves with varying degrees of antiparallel alignment according to the thickness of the spacer layer.

Why does this occur? It's not yet completely understood, although there has been a great deal of theorizing since these oscillations were discovered. Parkin believes the magnetic materials create about a 10-angstrom-wavelength, spinpolarization wave that extends all the way across the nonmagnetic layer. The orientation of the next magnetic layer would then depend on the intensity of this wave at the interface, which would be 
from page 116 combination of materials that gives a consistent and large GMR effect for very low external field strengths and that can also be manufactured reliably at relatively low cost.

'That's why it's so important to understand the science,' Parkin adds. 'Our intensive studies of GMR and the sources of the scattering have enabled us to enhance considerably the performance of some lowfield spin valves for use in future sensors.'

Even after a promising structure is found, the challenges of incorporating it into a product are not over. To operate reliably in disk drives, the spin-valve materials must resist corrosion, high electrical currents and other degrading influences.

Of course, mass producing millions of films only a few atoms thick will be a special challenge. 'Our first thin-film MR recording head called for a 200-angstromthick film,' says IBM Fellow David A. Thompson. 'At that time [the early 1970s] we could make those films in the lab, but it was way beyond what had been done in manufacturing.' Although it was difficult to achieve at first, they're now making them routinely. The situation with spin valves today is similar, but the dimensions are much smaller.
'If IBM is successful with GMR sensors,' Parkin adds, 'a major factor in that achievement will be the fundamental insights gained by today's scientific investigations.'

Grünberg's research is already paying off, in the form of patent license revenues to his laboratory, which has permitted him to buy new sputtering equipment for his future research into GMR applications.

Fert is examining a number of spindependent phenomena that have grown in interest since the discovery of GMR, including current-perpendicular-to-theplane GMR by exploiting step-bunching during the sample preparation and spindependent Coulomb blockade effects appearing in tunnel junctions with metallic clusters in the insulating interlayer.

Parkin is also working to help make magnetic tunnel junctions a reality. Rapid progress suggests that these types of structures have the potential to be an excellent non-volatile magnetic storage cell which will allow magnetic memories much faster and denser than existing approaches. Of course this depends on whether or not they can be designed and produced economically. Answers to these questions will take several more years of study.

\section{from page 117 determined by the} thickness of the nonmagnetic film. Indeed, for $\mathrm{Co} / \mathrm{Cu}$ it is possible to prepare crystalline multilayers oriented along different crystal directions, and it has been observed that the oscillation periods depend upon the orientation - in good agreement with theoretical models.

While the oscillation periods can be related to the electronic structure of the spacer material, what is not yet explained is why the coupling strength between magnetic materials varies so consistently across the periodic table.

The predicted 2-angstrom oscillations were eventually seen in 1991 in samples consisting of an iron whisker, a chromium interlayer and a thin iron film by Bob Cellota's group at the National Institute for Standards and Technology in the U.S. and Steve Purcell and co-workers of Philips in Eindhoven, Netherlands. So perfectly formed were these materials - grown by MBE - that Grünberg says 'these are probably still the best samples that have ever been made in the context of coupling, demonstrating the ideal result.'

This article is a special update for Europhysics News of an article the author wrote for IBM's Research Magazine in 1992. Michael Ross, who works at IBM's research centre at Almaden, California contributed to the book A Field Guide for Science Writers, Oxford University Press, 1997.

\section{Theoretical Physics (N.10) Experimental Physics (N. 20)}

The INFN Fellowship Programme 1997-98 offers thirty positions for non italian citizens for research activity in theoretical or experimental physics.

Fellowships are intended for young post-graduates not more than 35 years of age at the time of deadline.

Each fellowship is granted for one year (which may start during the period from September to November 1998), and may be extended for a second year.

The annual gross salary is 30.000 .000 Italian Lire, plus travel expenses for round trip transportation from the home fellows to the I.N.F.N. Section or Laboratory. Lunch tickets are provided for work days.

Candidates should submit an application form and a statement of their research interests, including three letters of reference.

Applications should sent to I.N.F.N. not later than October 31, 1997.
Candidates will be informed by the end of April 1998 about the decisions taken by I.N.F.N.'s committee.

The successfull applicants may carry out their research activity in Italy, at any of the following Laboratories and Sections of I.N.F.N.:

National Laboratories of Frascati (Rome), National Laboratories of Legnaro (Padua), Southern National Laboratory (Catania) and National Gran Sasso Laboratories (L'Aquila).

\section{I.N.F.N. Sections in the Universities of:}

Turin, Milan, Padua, Genoa, Bologna, Pisa, Rome "La Sapienza“, Rome „Tor Vergata“, Naples, Catania, Trieste, Florence, Bari, Pavia, Perugia, Ferrara, Cagliari, Lecce and National Institute for Health (Rome).

Information, requests for application forms, and applications should be addressed to Personnel Office Followship Service, National Institute of Nuclear Physics 